\title{
"YEA" or "NAY" in Psychology Science: An Analysis of the Postmodern Nature of Performing Psychology
}

\author{
Xingguo Zhu ${ }^{1}$, Jianjun Yin $^{2} \&$ Tracy Harris ${ }^{3}$ \\ ${ }^{1}$ Associate Professor of Education in the Department of Educational Psychology, Nantong University, China \\ ${ }^{2}$ Professor of Education in the Department of Elementary and Early Childhood Education, Yangzhou University, \\ China \\ ${ }^{3}$ Associate Professor of Education in the Department of Elementary and Early Childhood Education, Jackson \\ State University \\ Correspondence: Jianjun Yin, Professor of Education in the Department of Elementary and Early Childhood \\ Education, Yangzhou University, China.
}

Received: November 30, 2017

doi:10.20849/aes.v2i4.263
Accepted: December 20, 2017 Online Published: December 25, 2017

URL: https://doi.org/10.20849/aes.v2i4.263

Research on the impact of performance evaluation on professional identity of rural teachers in Jiangsu Provincial College of philosophy and social science research project is a phased study based on the role of psychological empowerment (approval number 2016SJB880094).

\begin{abstract}
Since becoming an independent subject, psychology has portrayed itself as a strict science on the way to the development of natural science. Because of breaking away from the social cultural and historical background of human life, scientific psychology is being questioned. In the doubt, non scientific psychology which is closer to social life and can adapt to the human need emerges as the times require. Performance psychology developing from social therapy insists on a new cultural-performatory approach to understanding human life, making psychology research return to life and helping people to restart their development. Non-epistemology, non-diagnosis and non-adaptability are the essential embodiment of performing psychology from the natural science to the post-modern humanities.
\end{abstract}

Keywords: performance psychology, non scientific psychology, postmodern essence

\section{Introduction}

In the early 1970s, the psychologist Klaus Holzkamp taking Carle Marx's sociological theory as a starting point, criticized the narrowness of modern psychology and pointed out that the narrow psychology only considered history as a natural process of development, but not distinguished the development of natural history from the history of human activities. The history of human activity is a specific history of human social practice, and human beings are not only the leader in this historical development, but also the result (Helmut ,2009, p.30). Similarly, influenced by Marx's philosophy, psychologists, Newman and Holzman, also put forward a strong challenge to modern psychology, which advocates the scientific paradigm as the main research method. They believe that the study of psychology cannot be separated from the social, cultural and historical background of human existence. The study of human psychology cannot stay at a certain historical moment, but should run through the whole process of social development. The study of psychology should not only focus on the natural attributes of human beings, but should also pay more attention to the social development of human beings. On the basis of questioning, Newman and others put forward the performance theory, and then developed into an important post-modern psychological theory-Performance Psychology. The study of a new psychological theory is inseparable from the analysis of its essential characteristics. Only by grasping its essential characteristics comprehensively and accurately can we better understand and apply this theory.

With the crisis of western social psychology and the discussion of crisis, psychologists began to reflect on the mode of naturalistic Psychological Science. Gergen, as the representative of postmodern psychologists, even began to speak out against and questioned the scientific psychology. They pointed out the drawbacks of the naturalistic model. They believe that human psychology is different from the material studied by natural science. 
Human psychology is the product of history, and varies with time, place, culture and history. Human psychology lacks the relative stability of ordinary matter, so the causality model of natural science is not suitable for Psychology (Ye ,1999). Since the 90s of last century, the influence of Postmodernism in psychology has produced a trend of postmodernism psychology characterized by opposition to positivism and scientism. These postmodernism psychology trends all have a common characteristic, that is "non scientific". The non scientific nature is put forward according to the modern scientific psychology research. Although postmodern psychology, which includes the performance psychology, holds a critical position on modern psychology, especially the psychology of scientism, non science and scientism is not in a completely opposite position. To correctly understand the non scientific characteristics of performing psychology, it is necessary to discuss the non scientific connotation and its non scientific characteristics in the research of performing psychology.

\section{2. "Yea" and "Nay" in Psychology Science}

Since it has become an independent discipline, mainstream psychology has been a natural science. But the question of mainstream psychology as a natural science has never ceased. With the rise of post-modern culture, people are more and more interested in non mainstream psychology. Psychological research is required to be more closely related to social life and better suited to human needs. Psychology, which is characterized by non natural science, is often referred to as non scientific psychology. Non scientific psychology contends that the study of psychology should be living people rather than thought, the relationship between people but not the behavior, a story rather than a reason, is to create a new way of life rather than adapt to the existing mode of alienation ( $\left.{ }^{\text {Yue }}, 1999\right)$. Non scientific psychology is a resistance to scientific hegemonism, yearning for the return of human life in psychology research, and also a pursuit of the human psychological process that psychology can focus on human life, culture and historical structure. Non scientific psychology comes into being and develops with the challenge of scientism and psychology.

\subsection{The Birth of Non Scientific Psychology}

American psychologist, Samson, pointed out that psychology is impossible to get rid of the influence of social culture, ideology and values, psychology should abandon the kind of natural science to create ultra culture psychology to achieve a "paradigm" of the revolution (Ye, 1999). In fact, the naturalistic view of science is also a product of a particular historical culture. In recent years, more and more psychologists have become aware of the disadvantages of naturalistic view of science and have turned to the research model of social culture, which, in a sense, is to promote the non scientific research in psychology.

\subsection{1 "Yea" and "Nay" in Psychological Research}

For all psychological researchers, the development of psychology into a scientific discipline is no longer in doubt. Although there are many schools in the field of psychology today, the purpose of their research is always to search for the laws of human psychology, to solve the psychological problems of human beings and to improve the quality of human life, which makes psychology a well deserved scientific discipline. Nowadays, the controversy about psychological research is mainly focused on the methodology of specific research. In the west, there has been a dispute between naturalistic psychology and humanistic psychology, scientific psychology and non scientific psychology. These arguments are concentrated on the scientific and non scientific nature of psychological research methods. The scientific nature of psychological research refers to its naturalistic research orientation, which proposes to make psychology a real empirical science

and advocates that in the study of psychology, experiments and statistical measurements should be taken as the center, and those who can study them in this way will be preserved, otherwise they will be excluded. However, the psychology of humanism, which is opposite to scientism in the study of psychology, is strongly opposed, and puts forward the idea of "non scientific psychology". Non scientific psychology advocates the use of non scientific methods to study psychology, and these methods mainly include introspection, speculation and hypothesis deduction. Their features are not to be repeated as the only standard, and belong to the category of non-empirical methods. These methods were widely used before the birth of scientific psychology. However, the positivist philosophy of science, which has been in the dominant position in psychological research, denies the value of these methods. After a long period of time in which psychology became a discipline, the non scientific methods were abandoned by psychology. It was not until 1970s when scientific psychology met with unprecedented difficulties that people realized the important role of non scientific methods in the study of psychology. Some psychologists have improved the traditional non scientific methods and applied them to the study of psychology. Since then many achievements are inseparable from the application of non scientific methods. For example, Piaget's division of children's thinking development stage, Kolberg's theory of moral development stage, Gardiner's theory of multiple intelligences, and so on. In the psychology of performance, 
Newman regarded philosophy (thinking of learning) as the first step for human beings to go out of their loneliness and depression. Holzman thinks this is a philosophical thinking without philosophy ( $\left.{ }^{\text {Gergen }}, 2001\right)$. It implies the application of the philosophical methods which have been separated from psychology to the study of psychology, thus showing the importance of non scientific in the study of postmodern psychology.

\subsubsection{Postmodern Turn in Humanistic Psychology}

Although "non scientific psychology" is an important branch of postmodernism psychology, it has the same long-term ideological origin as mainstream psychology. It is the continuation of humanistic psychology tradition (Yue,1999). Early in the establishment of scientific psychology, there are two kinds of different approaches - one is Wundt's natural science psychology research orientation and the other is created by Brentano humanities psychology research orientation (Guo ,2007,p.23). Although the development of these two kinds of research orientations are throughout the western psychology, the psychology research orientation of natural science has been in a dominant position, and the orientation of natural science psychology tries to make scientific psychology among natural science. Since its establishment, humanistic psychology has been trying to oppose the view that human beings are regarded as objects in the study of psychology and equated human psychological phenomena with natural phenomena, emphasizing that the study of psychology should respect the value and dignity of human beings. Humanistic psychology holds that the basic feature of human mental activity is its purpose and significance. So, different from the natural science research object, humanistic psychology cannot be limited to look only to those problems of scientific standards, and should include human face moments of inner experience, such as dignity, value, love, courage and humor. The humanistic approach to psychological research has been strongly opposed by humanistic psychologists who believe that psychology should be concerned with human science rather than physical science. In research methods, psychology advocates humanistic orientation of humanistic psychology as the representative of the whole analysis method, which emphasizes the unity of personality, and the dynamic process in real situations in the functioning of the personality of the all-round analysis. It also advocates the use of phenomenological methods, that is, a direct approach to the subject of research and a method of reporting introspective nature. In brief, it is a direct description of a person's conscious experience (Guo, 2007, p.319). Later, due to the challenge of Postmodernism to humanistic psychology, there was a postmodern turn in humanistic psychology, which is mainly characterized by the rise of qualitative methods. It is a typical non scientific approach to quantitative methods in empirical research. Therefore, the post-modern development of humanism is also an important reason for the rise of non scientific in psychological research. It can also be said to be the continuation of humanistic psychology.

\subsection{The Main Connotation of Non Scientific Psychology}

Post modernism thinks that scientism has brought unprecedented development and independent to the psychology research in psychology. In the short span of more than 100 years after the independence of psychology, scientism has achieved far greater results than all the research achievements in the long past. But on the other hand, the prevalence of scientism in the study of treating human beings as machines or animals has caused many people to feel the loss of the meaning and spiritual value of life in modern Western societies. The emergence of non scientific psychology is the declaration of war against scientism in psychology. Its unscientific nature includes the following points:

First, oppose the theory of mechanistic and reductionism. Non scientific psychology holds that scientism psychology insists on the research orientation of naturalism and positivism, and blindly imitates the research model of Physical Science in the study, and makes the objective experiment method unique and absolute (Gao ,2001,p.198). The scientific psychology, which inevitably leads to the inhuman study of psychology and falls into the realm of the theory of mechanistic and reductionism. The emphasis of scientific psychology on objective experimental method can only separate the relation between the subject of study and the object being studied, and give psychologists the illusion that they are unrealistic, and that they determine the actual state of mental phenomena by purely objective observation and pure form theory without any influence.

Therefore, non scientific psychology advocates that psychological research should highlight the subject and subjective role of human beings, realize the unity of subjective and objective two paradigms, emphasize the value and significance of the study of human nature, freedom of choice, and potential human, and oppose the reductionism of mechanical and psychoanalysis.

Second, opposition to the methodology centered theory. The fundamental error of scientism in methodology lies in the treatment of human, human nature or human psychology of "objectification" processing. Because of the natural science methods blindly worship, scientism psychology gradually formed a "methodology centered theory" which led to the only legitimate choice of objectivism, reductionism, value neutrality principle and 
quantitative research as to obtain the knowledge of Psychology. It caused the subjectivity loss, individual loss and overall digestion effect in the study of scientific psychology. Therefore, non scientific psychology puts forward the opposition methodology central theory, and advocates adopting an open, compatible and comprehensive approach to the research methods

Third, promote the integration of qualitative research and quantitative research. For a long time, although the quantitative and qualitative researches in psychology research are two important methods, qualitative research has been accompanied by quantitative research. But under the influence of positivism, qualitative research method has gradually become marginalized, especially in the background dominated by scientific psychology, psychologists often ignore the value of the qualitative research. Even some humanistic psychologists worship quantitative methods in order to make their research conform to the quantitative requirements of mainstream psychology. However, after the rise of postmodernism, starting from the angle of opposing methodology centered theory, postmodern psychology has "Deconstructed" the quantitative research methods of the aura, dispels the superstition to the quantitative method, and makes the qualitative research method obtain new vitality. In humanistic psychology, the rise of qualitative methods can be regarded as an organic part of the postmodern turn. Non scientific psychology suggests that in the study, we should combine qualitative and quantitative methods, and think that psychology which is only quantitative research without qualitative research is a kind of incomplete psychology. The research of psychology should pay attention to the meaning and value of life, and should be compatible with the research methods and perspectives of scientism and humanism, which will contribute to the healthy development of psychology.

Fourth, it emphasizes the transformation from individual rationality to relational rationality and emphasizes the importance of individual independence from others. Postmodernism thinks that individualism has become the source of various problems in modern society and excessive attention to self often leads to indifference, contempt and even hostility to others, thus creating the opposition between individuals. Postmodernism also advocates the shift from individual rationality to public reason, from individual self to relational self. Gergen pointed out: "the postmodern concept of individual rationality is that there are serious problems...... Language is a system that depends on itself. It exists before the individual, and then dies out of the individual. Therefore, as a rational individual, it is better to draw on the existing format or proper discourse style than to participate in a system that already has its own. In this sense, individual reason is only a form of cultural participation......" (Gergen, 2001, p.10). Postmodern psychologists call for the study of psychology to change from individual rationality to relational rationality. Man is not an entity, but a relation. Therefore, it has become an important feature of postmodern psychology to emphasize the study of psychology from the angle of relation.

\section{The "No" of Performance Psychology}

The non scientific nature of psychological research is very important, the main reason is that compared with the natural science, it has a very special object of study: human psychology and consciousness. Human psychology and consciousness can not be directly perceived and measured, and the formation of human psychology is also influenced by historical culture. The particularity of the object of psychological research requires that the researcher should go beyond the general verifiable principle in the course of research, and put people's psychology in a broader social context. Performing psychology is, in fact, a non scientific psychology of understanding human life in an unscientific way. Performing psychology uses a developmental approach to interpret the laws of human psychology, and is committed to integrating theoretical and practical research into the areas of psychotherapy, education, and social change etc... The "non scientific" trend of performing psychology is mainly embodied in the following three aspects:

\subsection{Non Epistemology}

Positivism holds that scientific knowledge is the expression of the objective experiential world, and science as a large amount of written commonsense knowledge is the means we use to describe the world (Xia, 2006, p.487). Influenced by positivism, scientism psychology is often keen on the construction of some theories. In the process of construction, it first observes the object, records its relevant features, and then establishes the theory in turn, and then tests its theoretical expressions, as for those that do not conform with the observation theory to modify or replace.

Therefore, scientism psychology advocates a study with the purpose of grasping knowledge, and believes that human learning process should be a process of acquiring knowledge. The essence of this kind of learning and research is that there is a gap between the way the world exists and what people think about it. The world is independent of what people think about it, the reason why people cannot really grasp the law of development of things is that people cannot properly describe what the world is, so the study of psychological science is to use 
experience accumulated to bridge this gap. Positivism holds that the existence of objective truth is independent of what people think about it and whether or not research is correct is to see whether it is consistent with objective truth. Influenced by postmodern constructivism, holds that knowledge is constructed, and there is no objective criterion for measuring truth. Performance psychology advocates limitations and the truth of scientific knowledge, and argues that they can change with history, society, and circumstances.

Therefore, the study of psychology is not a knowledge inquiry activity in order to obtain the objective laws of psychological activities, but a revolutionary activity that is not based on knowledge of non-cognitive and non-dualism. On this basis, performing psychology put forward new ideas of learning, students learning should not be equal to the cultivation of cognitive ability, but should be through creative activities so that students can be in a recent development zone to develop themselves. In addition, the performing psychology also advocates non cognitive activities of a collective, and that science is not on the objective reality of the phenomenon, but a kind of social system. It is not a construction of a single person, but a collective enterprise. The characteristics of non epistemology are also manifested in its view that human psychological activities have a unique and rich nature, so it cannot be studied by the dualist epistemology of the separation of subject and object. Therefore, performance psychology advocates that the study of psychology must adopt a non epistemological approach to practice.

\subsection{Non Diagnosis}

The understanding of performing psychology is often associated with the application of social therapy in the field of psychotherapy. In fact, the emergence and development of performing psychology can not be separated from the study of the theory and practice of social therapy. The non scientific nature of performing psychology is its non diagnosis in the field of social therapy. Generally speaking, different pathological views can lead to different treatment views. The modern psychotherapy, which based on the psychology of scientism, often equated human mental problems with physical diseases in medicine. As a doctor diagnoses, modern psychotherapy is also the psychological diagnosis to visitors as a necessary step, and also announced a unified diagnosis standard in the field of psychotherapy, which leads to all psychological problems being posted on various labels in psychotherapy. The "non" of performing psychology in traditional psychotherapy is reflected in the question of modern psychotherapy, especially the diagnostic process, and the concept of non diagnosis is put forward.

The diagnosis is very important for a serious disease in the physiological aspects of patients. But for those who are suffering from social problems and who have slight psychological problems, the diagnosis cannot help the psychological treatment, and even makes the labeled visitors suffer some psychological burden. In addition, for therapists, psychological diagnosis by a certain standard will not only limit the therapist's perspective, but also affect the therapist's ability to treat the client as a person to establish a good doctor-patient relationship. Furthermore, different from the various physical phenomena in nature which have stable properties, because the human mind is unstable and changeable, people, once the so-called diagnosis is made, tend to selectively ignore some of the very symptoms that the patient does not fit into the diagnosis, and over pay attention to the symptoms that are consistent with the initial diagnosis. In this way, diagnosis will not only cause psychological pressure to visitors, but also infiltrate the negative influence into the life of the future, which will lead to more psychological problems.

Performance psychology regards the diagnosis in psychotherapy as a breakthrough point in criticizing and changing modern psychotherapy. After fully analyzing the disadvantages of modern psychotherapy, especially the diagnosis, performing psychology definitely puts forward the concept of non diagnosis. Non diagnosis is not only a challenge to the application of traditional psychology in the field of psychotherapy, but also the application of Wittgenstein's theory of relational activity in the field of postmodern psychotherapy.

Social treatment is committed not to digging the visitors' real life, but to helping people's creative performance to change the original form of life and restart their new life.

\subsection{Non Adaptability}

The study of natural science is to reveal the universal objective law of the development of things, and only by following this law can man and society develop. Attention theory reflected in the field of psychology research is to find out the law of development of human psychology and thinking, for those people who have problems to be treated, they can eventually adapt to the social environment, whether the environment is adapted to human growth or not. Performance psychology believes the idea of adapting people to the environment is passive and negative, which cannot help people grow and develop, and hinder the development of human being to a certain extent. In order to oppose how to help people adapt to environment negatively in modern psychological research, 
Newman, Holzman and others follow Marx revolution thought, seek to take the method and purpose as a unity, and regard the unity as a way of social life and learning life. The study of performing psychology is concerned with the attempt to abandon a knowledge based epistemology and seek a revolutionary form of performance (Kopano, 2006). The revolutionary practice advocated by Newman and others is aimed at the traditional adaptive activities. Over the past thirty years, their practice of developing performance as the main form has gone beyond their original expectations (Newman \& Holzman, 1996, p.viii). The developing communities they have created have attracted thousands of people from all over the world. For their own development, they are no longer confined to passive and passive life, but participate in all kinds of performances actively. Only from the increasing number of participants can we see that the development of performance advocated by performing psychology is undoubtedly successful in promoting the growth and development of people.

\section{4. "Yes" and "No" in Performing Psychology}

The scientific and non scientific aspects of psychological research exist at the same time. "Non scientific" is a breakthrough of scientism psychology which takes the method as the center and the research orientation. Modern psychology often uses the empirical method in the course of the research to judge whether it is scientific basis, which limits the methodological innovation in the study of Psychology. Non scientific psychology advocates the unity of methods and objects, the unity of methods and theories, promotes the diversification of research methods and a breakthrough in the methodological centralization of scientific psychology, which is the non scientific embodiment of postmodern psychology, including performing psychology. Because people cannot accurately understand and grasp the essence of non science, there are always two misunderstandings: on the one hand, people think that the study of non scientific psychology, which aims at opposing positivism research methods, will make psychological research go back to the old way of philosophical thinking, and will become a counter flow of science, thus hindering the development of psychology. On the other hand, people think that non science and science are opposite, the relationship is either this or that. In fact, these two kinds of knowledge are incorrect. The non scientific psychology only deny the thought that scientism has overweening status, but not completely deny the role of scientism in psychological research. In the view of postmodernism, whether it is from determinism to emergentism, or from reductionism to pluralism, science is undergoing unprecedented changes, followed by a variety of traditional integration and a variety of perspectives coexist. Both scientific and non scientific viewpoints are only one of many views, because one view alone cannot accommodate the increasingly pluralistic orientation of psychological research. Therefore, the relationship between science and non science in psychological research should be dialectically analyzed, rather than simply put them in opposition. Scientism psychology plays an irreplaceable role in the development of psychology, but scientific psychology alone is not enough to explain the complex psychological problems of human beings.

Emphasizing the non scientific nature of psychological research does not mean the denial of science. The weakness of scientific methods in the study of psychology is not the empirical method itself, but this method which is not suitable for all psychological research. The scientific methods in psychological research are consistent with the purpose of non scientific methods, and should be complementary in practical application (Shi, 2001). Thus, the scientific and non scientific nature of performing psychology should also be a complementary relationship, rather than opposing each other. Therefore, in the study of psychology, we should not only pay attention to its scientific nature, but also pay attention to its non scientific nature. Only by integrating the two into the study of psychology can we benefit from the progress and development of Psychology.

\section{References}

Benyu, Guo. (2007). History of Western Psychology. Beijing: People's Medical Publishing House.

Fengqiang, Gao. (2001). The Dilemma and Outlet of Modern Psychological Paradigm: a Study of Postmodern Psychological Thought. Beijing: People's publishing house.

Gergen, K. (2001). Psychological Science in the Postmodern Context. American Psychologists, 56(10), 10-34. https://doi.org/10.1037/0003-066X.56.10.803

Guoan, Yue, \& Qi, Hao. (1999). Theory and Method of Social Therapy: a Review of the Treatment of Postmodern Psychology. Nankai Journal, 6, 86-91.

Haosheng, Ye. (1999). On the Three Turn of Modern Psychology. Jianghai Academic Journal, 3, 99-10.

Helmut E. Luck. (2009). The history of psychology, translated by Lv Na et al.. Shanghai Academic Press.

Jisong, Xia. (2006). Modern Western Philosophy. Shanghai: Shanghai People's Publishing House.

Kopano, Ratele. (2006). Book Reviews of Postmodern Psychologies, Societal Practice, and Political Life. South 
African Journal of psychology, 36(2), 446-452.

Newman, F., \& Holzman, L. (1996). Unscientific Psychology: A Cultural-Performatory Approach to Understanding Human Life. Westport, Connecticut and London: Praeger Publishers.

Tieru, Shi. (2001). The Speculative Method in Psychological Research. Journal of Southern China Normal University.

\section{Copyrights}

Copyright for this article is retained by the author(s), with first publication rights granted to the journal.

This is an open-access article distributed under the terms and conditions of the Creative Commons Attribution license (http://creativecommons.org/licenses/by/4.0/). 